\title{
Comportamento de Índices de Diversidade na Composição da Arborização de Ruas
}

\author{
Rogerio Bobrowski ${ }^{1}$, Daniela Biondi ${ }^{2}$ \\ ${ }^{1}$ Universidade Estadual do Centro-Oeste - UNICENTRO, Irati/PR, Brasil \\ ${ }^{2}$ Universidade Federal do Paraná - UFPR, Curitiba/PR, Brasil
}

\begin{abstract}
RESUMO
Nesta pesquisa analisou-se o comportamento de índices de diversidade frente a diferentes cenários de composição com riqueza de espécies e uniformidade distintas, a influência do espaçamento de plantio na determinação dos valores desses índices e o comportamento dos dados de diversidade de um inventário da arborização em relação a dois cenários de composição aproximados aos dados reais. Constatou-se que há influência significativa do aumento do espaçamento de plantio somente para os índices de riqueza específica. Houve diferença significativa entre os valores de todos os índices para os cenários de diversidade elaborados. Nos cenários analisados, os índices de riqueza específica apresentaram valores menores e mais estáveis que os do inventário, e os índices de equidade apresentaram valores maiores.
\end{abstract}

Palavras-chave: floresta urbana, silvicultura urbana, ecologia urbana, paisagismo.

\section{Diversity Indexes Performance in the Tree-lined Street Composition}

\begin{abstract}
In this research was analyzed the performance of diversity indexes facing different scenarios of composition with distinct species richness and uniformity, the influence of planting spacing in the determination of values of these indexes and the behavior of diversity data of a street tree inventory in relation to two scenarios of composition approximated to real data. It was noted that there is significant influence of increased planting spacing only for species richness indexes. There was significant differences between values of all indexes for the diversity scenarios elaborated. In the scenarios analyzed, the species richness indexes showed lower and more stable values than inventory ones and the evenness indexes showed higher values.
\end{abstract}

Keywords: urban forest, urban forestry, urban ecology, landscaping. 


\section{INTRODUÇÃO}

A diversificação de espécies na arborização de ruas está dentre os principais objetivos do processo de gestão do componente arbóreo nas cidades (Nagendra \& Gopal, 2010; Kenney et al., 2011; Bobrowski \& Biondi, 2012), primordialmente para manter a diversidade biológica e a diversidade genética, pois essas determinam a estabilidade, a resistência e a resiliência diante de adversidades (Galvin, 1999; Raupp et al., 2006; Teixeira et al., 2011). De acordo com Sreetheran et al. (2011), o exame da diversidade ocorrente na arborização de ruas permite, de forma mais eficiente, a gestão de estratégias de plantio. Para Jim \& Chen (2009), investigações sobre a composição de espécies, abundância e distribuição passam a ser estratégicas na conservação da biodiversidade na arborização de ruas, porém isto é desafiador para os países em desenvolvimento.

Há grande preocupação com a diversidade de espécies nas cidades (Biondi \& Leal, 2008), por ser um dos atributos de qualificação da sustentabilidade da arborização urbana (Kenney et al., 2011) e por visar a redução das perdas ocasionadas por pragas e doenças, bem como o prolongamento dos benefícios proporcionados pelas árvores (Galvin, 1999; Raupp et al., 2006; Teixeira et al., 2011). Apesar de a diversidade ser traduzida como preocupação com a riqueza e equidade de espécies, a adaptabilidade delas às condições de estresse urbano é que deveria ser mais importante como objetivo do planejamento (Richards, 1993; Raupp et al., 2006).

Mesmo atendendo a proposições subjetivas, a baixa proporção observada para algumas espécies não consegue eliminar os problemas, pois muitas pragas podem atacar mais de uma espécie (Raupp et al., 2006). Desta forma, Sjöman et al. (2012) afirmaram que ainda não há conhecimento apropriado e experiências disponíveis sobre níveis de diversidade sustentáveis para árvores urbanas.

Com relação às experiências de cidades brasileiras e de outros países, alguns inventários conduzidos utilizaram índices de diversidade para caracterizar a sua sensibilidade à arborização de ruas e para descrever o comportamento da composição e diversidade de espécies (Thompson et al.; 2004; Silva \& Bortoleto, 2005; Jim \& Chen, 2009; Nagendra \& Gopal, 2010;
Sreetheran et al., 2011; Subburayalu \& Sydnor, 2012; Cowett \& Bassuk, 2014). Nas justificativas de uso ou descrições metodológicas desses índices é comum não ser informado qual o objetivo de mensuração do índice utilizado: se riqueza específica, se abundância ou se equidade, conforme as definições apresentadas por Moreno (2001) e Magurran (2011)

Segundo Moreno (2001), pode-se utilizar índices de diversidade para a análise de ecossistemas tanto naturais quanto alterados, com a intenção de se encontrar padrões que sejam capazes de oferecer estimativas confiáveis de diversidade biológica. Silva \& Bortoleto (2005) afirmaram que esses índices podem ser empregados como ferramenta do manejo e do plano diretor da arborização urbana. Entretanto, Insernhagen et al. (2009) argumentaram que a divulgação de valores de índices de diversidade deve ser feita com cautela, porque eles podem mascarar a presença de espécies exóticas e exóticas invasoras, o que pode não indicar boa qualidade ambiental da arborização.

A hipótese lançada para esta pesquisa foi a de que é possível obter valores ideais hipotéticos de diversidade para a arborização de ruas, ajudando na interpretação dos dados obtidos nos inventários florestais. Portanto, os objetivos foram analisar o comportamento de índices de diversidade frente a diferentes cenários de composição, analisar a influência do espaçamento de plantio na determinação dos valores desses índices e analisar o comportamento dos valores de diversidade de um inventário da arborização em relação a dois cenários de composição com semelhanças em relação aos dados reais.

\section{MATERIAL E MÉTODOS}

Esta pesquisa foi realizada a partir de informações de um inventário de reamostragem da arborização de ruas realizado na cidade de Curitiba, Paraná, no ano de 2010, por Bobrowski (2011). Curitiba está localizada na região leste do estado, no primeiro planalto paranaense, entre as coordenadas $25^{\circ} 25^{\prime} 48^{\prime \prime} \mathrm{S}$ e $49^{\circ} 16^{\prime} 15^{\prime \prime} \mathrm{O}$ (Bobrowski, 2011).

A partir dos croquis de localização de campo foram delimitadas as 15 unidades amostrais de $500 \times 500 \mathrm{~m} \mathrm{em}$ um sistema de informação geográfica (SIG), tomando por base os pontos e distâncias de amarração constantes nesses documentos e a base cartográfica do município. 
Todas as variações de forma dos quadrados amostrais que resultavam de erros analógicos dos croquis foram desprezadas para que se pudesse obter o mesmo valor de área em todas as unidades amostrais e evitar tendenciosidade na estimativa da diversidade a partir dos cenários de diversidade elaborados.

Para a criação desses cenários foi necessária a obtenção de informações a respeito dos espaçamentos médios de plantio, no inventário da arborização, e do comprimento das calçadas nos trechos de rua inseridos nas unidades amostrais no SIG. As classes de espaçamento adotadas foram definidas com base no dobro da média do maior valor do raio de copa mensurado para cada uma das espécies, com árvores remanescentes entre a primeira e a segunda ocasião de medição. Isso posto para indicar o espaçamento mais adequado, no qual as copas se tocariam sem se entrelaçar. As classes definidas foram: $4 \mathrm{~m}, 6 \mathrm{~m}, 8 \mathrm{~m}$, $10 \mathrm{~m}, 12 \mathrm{~m}$ e $14 \mathrm{~m}$. Para cada parcela inserida em ambiente SIG foram obtidas medidas do comprimento total de calçadas, de cada lado das ruas inseridas na unidade amostral, sendo descontada a distância de $5 \mathrm{~m}$ para cada esquina, como fator de segurança adotado na implantação da arborização de ruas e recomendado nos manuais de arborização e planos diretores da arborização urbana.

Com os valores de comprimento de calçada e com as classes de espaçamento estabelecidas foi estimada a quantidade de árvores para cada rua das unidades amostrais, em ambas as calçadas. A partir disso foram planejados 10 cenários de diversidade considerando diferentes composições florísticas em termos de riqueza específica e uniformidade de composição, conforme descrições a seguir:

Cenário 1: Uma espécie por parcela;

Cenário 2: Duas espécies por parcela, sendo uma para cada perfil de rua;

Cenário 3: Uma espécie para cada rua da parcela (repetindo nas 15 parcelas);

Cenário 4: Duas espécies para cada rua da parcela (repetindo nas 15 parcelas), sendo uma para cada perfil de rua;

Cenário 5: Uma espécie para cada rua da parcela (sem repetir nas 15 parcelas);
Cenário 6: Duas espécies para cada rua da parcela (sem repetir nas 15 parcelas), sendo uma para cada perfil de rua;

Cenário 7: Duas espécies por parcela, em ruas transversais (sem repetir nas 15 parcelas);

Cenário 8: Quatro espécies por parcela, em ruas transversais (sem repetir nas 15 parcelas), sendo uma para cada perfil de rua;

Cenário 9: Quatro espécies por parcela, em ruas transversais (sem repetir nas 15 parcelas);

Cenário 10: Oito espécies por parcela, em ruas transversais (sem repetir nas 15 parcelas), sendo duas para cada perfil de rua (alternadas em ruas transversais).

Para cada cenário de diversidade e para o inventário florestal foram determinados os valores de índices que expressam a diversidade de espécies, em quatro categorias distintas, conforme definições feitas por Moreno (2001) e Magurran (2011). Os índices utilizados foram: Margalef e Meinhinick (riqueza específica), Simpson e McIntosh (dominância), Bulla e Pielou (equidade) e Shannon-Wiener (informação).

A influência do espaçamento e da composição dos cenários sobre os valores dos índices foi analisada tomando-se por base os valores obtidos nas unidades amostrais. As análises foram realizadas por meio do teste de Kruskal-Wallis (Siegel \& Castellan, 2006), via software R, com uso do pacote Stats (R Development Core Team, 2014). Quando pertinente, foi aplicado um teste post hoc, conforme sugerido por Siegel \& Castellan (2006), para identificação de diferenças significativas entre as médias, mediante uso do pacote PMCMR (Pohlert, 2014), ao nível de 5\% de significância.

Também foi realizada análise de correlação entre os valores dos índices, tanto daqueles obtidos nos cenários de diversidade quanto daqueles obtidos no inventário florestal, a fim de verificar a natureza da relação, se direta ou inversa.

Com relação ao índice de Shannon-Wiener, foi realizada a análise SHE (Magurran, 2011) para compreender a influência das componentes riqueza específica (S) e uniformidade (E) sobre os valores do índice, tanto para os dados dos cenários de diversidade quanto para os dados do inventário florestal. 


\section{RESULTADOS E DISCUSSÃO}

A quantidade de árvores estimadas para os espaçamentos hipotéticos de plantio $(4 \mathrm{~m}, 6 \mathrm{~m}, 8 \mathrm{~m}$, $10 \mathrm{~m}, 12 \mathrm{~m}$ e $14 \mathrm{~m}$ ) foram respectivamente iguais a 19.847, 13.231, 9.923, 7.939, 6.616 e 5.670 árvores. Em cada cenário de diversidade analisado (Tabela 1) foram considerados esses valores para as estimativas de diversidade. Deve-se destacar que para uma situação real de plantio não seria possível estabelecer com exatidão espaçamentos equidistantes devido à variação espacial de localização de estruturas urbanas, como as guias rebaixadas, postes, caixas de inspeção de tubulações, redes de energia subterrâneas, dentre outras. Portanto, os valores de espaçamento indicados devem ser considerados como valores médios para fins de interpretação e análise comparativa com dados de inventários da arborização de ruas.

A análise dos dados do inventário demonstrou que o espaçamento médio das árvores foi de aproximadamente $12 \mathrm{~m}$ (coeficiente de variação médio de $88,72 \%$ ), variando de $9,60 \mathrm{~m}$ a $18,30 \mathrm{~m}$ entre as unidades amostrais. A amplitude do coeficiente de variação (de 63,41\% a 120,47\%) expressa a própria variabilidade de localização das estruturas urbanas (guias rebaixadas, postes, esquinas, dentre outros) que condicionam o plantio das árvores e limitam a adoção de espaçamentos regulares ao longo de uma calçada. Da mesma forma, é resultante da

Tabela 1. Número total e médio de espécies estimadas para cada cenário de diversidade.

Table 1. Total and mean number of species estimated for each diversity scenario.

\begin{tabular}{|ccc|} 
Cenário & $\begin{array}{c}\text { Número total de } \\
\text { espécies }\end{array}$ & $\begin{array}{c}\text { Número médio de } \\
\text { espécies }\end{array}$ \\
\hline 1 & 15 & 1 \\
\hline 2 & 30 & 2 \\
\hline 3 & 15 & 11 \\
\hline 4 & 29 & 22 \\
\hline 5 & 170 & 11 \\
\hline 6 & 331 & 22 \\
\hline 7 & 30 & 2 \\
\hline 8 & 60 & 4 \\
\hline 9 & 119 & 8 \\
\hline 10 & 236 & 16 \\
\hline
\end{tabular}

variabilidade de espaçamento condicionado pelos plantios irregulares (voluntários) feitos pela população (Bobrowski \& Biondi, 2012), o que contribui com a introdução de árvores muito próximas umas das outras. Quanto à quantidade de árvores e espécies amostradas no inventário observaram-se 4.360 árvores e 122 espécies diferentes.

Tomando por base a informação do espaçamento médio (12 m), a quantidade de árvores (4.360 árvores) e a quantidade de espécies e árvores mensuradas no inventário (122 espécies), o cenário que melhor descreve a situação encontrada, em relação à diversidade, é o cenário 9 (Tabela 1). Entretanto, se considerado o número médio de espécies por unidade amostral do inventário (31), a correspondência mais aproximada seria dos cenários 4 e 6 .

Para os dados analisados foi constatado que há diferença significativa entre as classes de espaçamento para o índice de Margalef $\left(\chi^{2}=13,78\right.$; $\mathrm{GL}=5$; $p$-valor $=0,017)$ e para o índice de Menhinick $\left(\chi^{2}=55,83 ; \mathrm{GL}=5 ; p\right.$-valor $\left.=8,79^{-11}\right)$, quando utilizados os valores de riqueza obtidos em cada unidade amostral a partir do número de espécies e de árvores correspondentes. A análise dentro de cada cenário também demonstrou diferença significativa ( $p$-valor $<0,05)$ e sensibilidade dos índices de riqueza específica à variação do espaçamento (Tabela 2). Os valores encontrados para cada cenário aumentam proporcionalmente com o aumento do valor do espaçamento (Figura 1).

Nesse caso, a redução da quantidade de árvores na composição florística do cenário enfatiza a importância do número de espécies, aumentando o valor do índice de riqueza. Isso ocorre porque os índices de riqueza de Margalef e de Menhinick assumem uma relação direta entre o número de espécies e o número de indivíduos amostrados (Moreno, 2001; Magurran, 2011). Portanto, a análise comparativa de dados da arborização de ruas, entre cidades ou bairros, deve ser feita com cautela, tomando cuidado para que os espaçamentos médios de plantio sejam iguais ou o mais próximo possível. Isso é necessário porque a variação ou irregularidade do espaçamento entre árvores, por restrições da estrutura urbana, remoção de árvores ou inexistência de plantio, pode ser diferente entre os locais em comparação, o que 
Tabela 2. Valores médios dos índices de riqueza específica, por espaçamento (Esp) e em cada cenário (Cen), a partir dos dados das 15 unidades amostrais.

Table 2. Mean values of species richness indexes, by spacing (Esp) and in every scenario (Cen), from data of fifteen unit plots. Means followed by the same letter in columns have no significative difference $(p>0.05)$.

\begin{tabular}{|c|c|c|c|c|c|c|c|c|c|c|}
\hline \multirow{2}{*}{ Esp } & \multicolumn{10}{|c|}{ Índice de Margalef } \\
\hline & Cen 1 & Cen 2 & Cen 3 & Cen 4 & Cen 5 & Cen 6 & Cen 7 & Cen 8 & Cen 9 & Cen 10 \\
\hline $4,00 \mathrm{~m}$ & --- & $0,14 \mathrm{~d}$ & $1,44 \mathrm{~b}$ & $2,93 b$ & $1,44 \mathrm{~b}$ & $2,93 b$ & $0,14 \mathrm{~d}$ & $0,42 \mathrm{~d}$ & $0,97 d$ & $2,05 \mathrm{~d}$ \\
\hline $6,00 \mathrm{~m}$ & --- & $0,15 \mathrm{~cd}$ & $1,52 \mathrm{~b}$ & $3,11 \mathrm{~b}$ & $1,52 \mathrm{~b}$ & $3,11 \mathrm{~b}$ & $0,15 \mathrm{~cd}$ & $0,44 \mathrm{~cd}$ & $1,02 \mathrm{~cd}$ & $2,17 \mathrm{~cd}$ \\
\hline $8,00 \mathrm{~m}$ & --- & $0,15 b c$ & $1,59 b$ & $3,24 \mathrm{~b}$ & $1,59 b$ & $3,24 \mathrm{~b}$ & $0,15 b c$ & $0,46 b c$ & $1,07 b c$ & $2,27 b c$ \\
\hline $10,00 \mathrm{~m}$ & --- & $0,16 \mathrm{ab}$ & $1,65 b$ & $3,36 \mathrm{~b}$ & $1,65 b$ & $3,36 \mathrm{~b}$ & $0,16 \mathrm{ab}$ & $0,48 \mathrm{ab}$ & $1,11 \mathrm{abc}$ & $2,35 \mathrm{abc}$ \\
\hline $12,00 \mathrm{~m}$ & --- & $0,16 a$ & $1,70 \mathrm{~b}$ & $3,46 \mathrm{~b}$ & $1,70 \mathrm{~b}$ & $3,46 \mathrm{~b}$ & $0,16 \mathrm{a}$ & $0,49 a$ & $1,14 \mathrm{ab}$ & $2,42 \mathrm{ab}$ \\
\hline $14,00 \mathrm{~m}$ & --- & $0,17 \mathrm{a}$ & $1,74 \mathrm{a}$ & $3,55 \mathrm{a}$ & $1,74 \mathrm{a}$ & $3,55 \mathrm{a}$ & $0,17 \mathrm{a}$ & $0,51 \mathrm{a}$ & $1,17 \mathrm{a}$ & $2,49 \mathrm{a}$ \\
\hline \multirow{2}{*}{ Esp } & \multicolumn{10}{|c|}{ Índice de Menhinick } \\
\hline & Cen 1 & Cen 2 & Cen 3 & Cen 4 & Cen 5 & Cen 6 & Cen 7 & Cen 8 & Cen 9 & Cen 10 \\
\hline $4,00 \mathrm{~m}$ & --- & $0,06 \mathrm{~d}$ & $0,31 \mathrm{~d}$ & $0,61 d$ & $0,31 \mathrm{~d}$ & $0,61 \mathrm{~d}$ & $0,06 \mathrm{~d}$ & $0,11 \mathrm{~d}$ & $0,22 \mathrm{~d}$ & $0,43 \mathrm{~d}$ \\
\hline $6,00 \mathrm{~m}$ & --- & $0,07 \mathrm{~cd}$ & $0,38 \mathrm{~cd}$ & $0,74 \mathrm{~cd}$ & $0,38 \mathrm{~cd}$ & $0,74 \mathrm{~cd}$ & $0,07 \mathrm{~cd}$ & $0,14 \mathrm{~cd}$ & $0,27 \mathrm{~cd}$ & $0,53 \mathrm{~cd}$ \\
\hline $8,00 \mathrm{~m}$ & --- & $0,08 b c$ & $0,44 b c$ & $0,86 \mathrm{bc}$ & $0,44 b c$ & $0,86 \mathrm{bc}$ & $0,08 \mathrm{bc}$ & $0,16 \mathrm{bc}$ & $0,31 \mathrm{bc}$ & $0,61 b c$ \\
\hline $10,00 \mathrm{~m}$ & --- & $0,09 \mathrm{ab}$ & $0,49 \mathrm{ab}$ & $0,96 a b$ & 0,49ab & $0,96 \mathrm{ab}$ & $0,09 \mathrm{ab}$ & $0,17 \mathrm{ab}$ & $0,35 \mathrm{ab}$ & $0,69 \mathrm{ab}$ \\
\hline $12,00 \mathrm{~m}$ & --- & $0,10 \mathrm{a}$ & $0,54 \mathrm{ab}$ & $1,05 \mathrm{ab}$ & $0,54 \mathrm{ab}$ & $1,05 \mathrm{a}$ & $0,10 \mathrm{a}$ & $0,19 a$ & $0,38 \mathrm{a}$ & $0,75 a$ \\
\hline $14,00 \mathrm{~m}$ & --- & $0,10 \mathrm{a}$ & $0,58 \mathrm{a}$ & $1,14 \mathrm{a}$ & $0,58 \mathrm{a}$ & $1,14 \mathrm{a}$ & $0,10 \mathrm{a}$ & $0,21 \mathrm{a}$ & $0,41 \mathrm{a}$ & $0,81 \mathrm{a}$ \\
\hline
\end{tabular}

Médias seguidas pela mesma letra na coluna não diferem significativamente $(p>0,05)$.

(a)
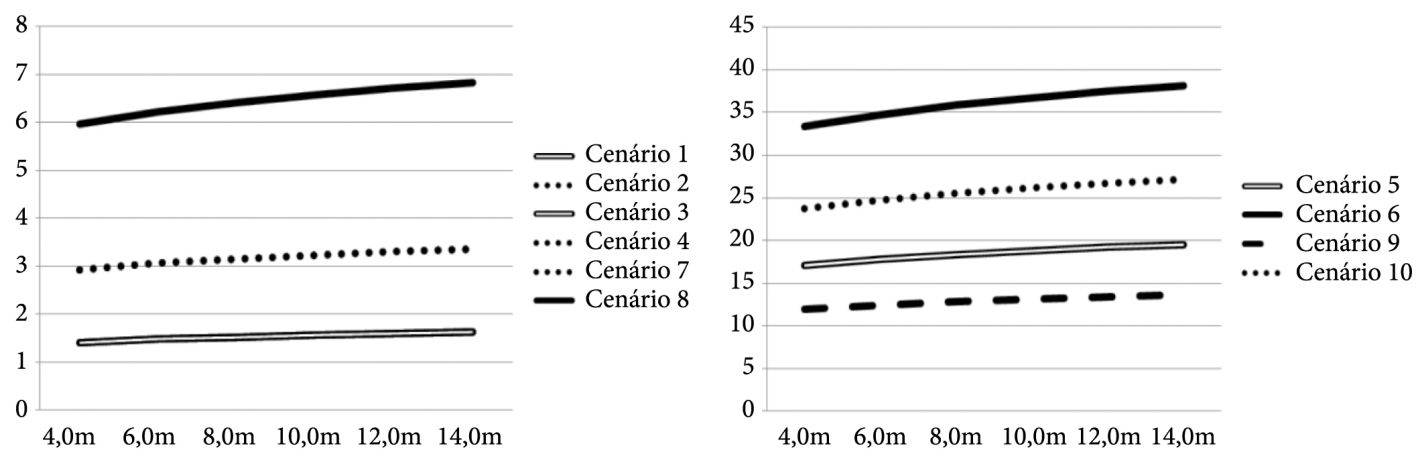

(b)
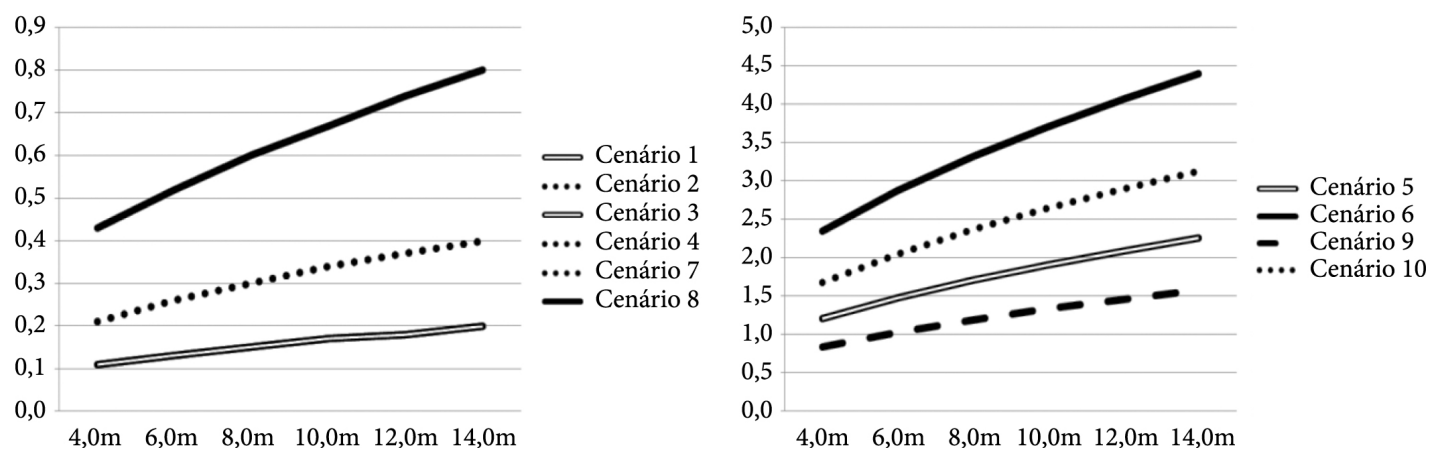

Figura 1. Variação dos índices de riqueza específica de Margalef (a) e de Menhinick (b) em função do espaçamento, obtidos do valor total da riqueza específica das 15 unidades amostrais.

Figure 1. Variation of species richness indexes of Margalef (a) and Menhinick (b) as a function of spacing, obtained from total values of species richness of fifteen unit plots. 
se reflete em espaçamentos médios distintos e na estimativa particularizada da riqueza específica.

A análise comparativa entre os diferentes cenários demonstrou que há diferença significativa entre eles em relação ao índice de Margalef $\left(\chi^{2}=773,10\right.$; $\mathrm{GL}=8 ; p$-valor $\left.<2,26^{-16}\right)$ e ao índice de Menhinick $\left(\chi^{2}=729,76 ; \mathrm{GL}=8 ; p\right.$-valor $\left.<2,26^{-16}\right)$. Entretanto, quando detalhada a comparação por meio do teste post hoc verificou-se que não há diferença significativa entre alguns cenários (Tabela 3). O cenário 1 foi excluído da análise por não apresentar variação da riqueza específica e incorrer em erros que impediram a análise estatística. Dentre essas semelhanças entre cenários destacam-se a entre os cenários 2 e 7 , a entre os cenários 3 e 5, a entre os cenários 4 e 6 e a entre os cenários 8 e 9 .

Outro fato importante a considerar sobre esses índices é que, apesar de serem uma forma de expressão da diversidade com uma tentativa de compensação do esforço amostral, eles são influenciados pela quantidade de amostras obtidas para análise e pela área mínima estabelecida por unidade amostral. Dessa forma, qualquer análise comparativa de informações obtidas em locais distintos deve ser feita adotando-se a rarefação dos dados, a fim de ponderar as diferenças decorrentes de intensidades amostrais diferentes, desde que a técnica de amostragem seja a mesma (Magurran, 2011). Isso deve ser levado em consideração, pois afeta a quantidade de árvores amostradas.

Por exemplo, Richter et al. (2012) obtiveram o valor de 26 para o índice de Margalef para o levantamento feito nas vias públicas da cidade de Mata, RS. Todavia, não foram dadas informações sobre o espaçamento médio entre as árvores, tampouco sobre a área amostrada e a intensidade amostral. Miranda \& Carvalho (2009) encontraram o valor de 25,9 para esse índice ao analisar a arborização do Bairro Ronda, em Ponta Grossa, PR, por meio de censo arbóreo. No trabalho apresentado não indicaram a área do bairro amostrado, mas apenas o número de vias públicas avaliadas. Diante destas informações, outro fator a considerar nas análises comparativas é que as estimativas de riqueza específica podem ser feitas a partir do número total de espécies e de indivíduos do inventário ou a partir dos valores observados para essas duas variáveis em cada unidade amostral (Schaaf et al., 2006; Kanieski et al., 2010).

Tabela 3. Valores de significância estatística ( $p$-valor) para a análise comparativa entre os valores médios de riqueza específica dos cenários de diversidade testados.

Table 3. Statistical significance values ( $p$-value) for the comparative analysis among average values of species richness of diversity scenarios tested.

\begin{tabular}{|c|c|c|c|c|c|c|c|c|}
\hline \multicolumn{9}{|c|}{ Cenários analisados pelo índice de Margalef } \\
\hline & 2 & 3 & 4 & 5 & 6 & 7 & 8 & 9 \\
\hline 3 & $7,7^{-14}$ & & & & & & & \\
\hline 4 & $<2,0^{-16}$ & $1,4^{-12}$ & & & & & & \\
\hline 5 & $7,7^{-14}$ & 1,000 & $1,4^{-12}$ & & & & & \\
\hline 6 & $<2,0^{-16}$ & $1,4^{-12}$ & 1,000 & $1,4^{-12}$ & & & & \\
\hline 7 & 1,000 & $7,7^{-14}$ & $<2,0^{-16}$ & $7,7^{-14}$ & $<2,0^{-16}$ & & & \\
\hline 8 & 0,004 & $2,6^{-9}$ & $<2,0^{-16}$ & $2,6^{-9}$ & $<2,0^{-16}$ & 0,0035 & & \\
\hline 9 & $3,0^{-9}$ & 0,003 & $<2,0^{-16}$ & 0,003 & $<2,0^{-16}$ & $3,0^{-9}$ & 0,1766 & \\
\hline 10 & $<2,0^{-16}$ & 0,004 & 0,006 & 0,004 & 0,006 & $<2,0^{-16}$ & $8,3^{-14}$ & $4,3^{-13}$ \\
\hline \multicolumn{9}{|c|}{ Cenários analisados pelo índice de Menhinick } \\
\hline 3 & $7,8^{-14}$ & & & & & & & \\
\hline 4 & $<2,0^{-16}$ & $1,7^{-10}$ & & & & & & \\
\hline 5 & $7,8^{-14}$ & 1,000 & $1,7^{-10}$ & & & & & \\
\hline 6 & $<2,0^{-16}$ & $1,7^{-10}$ & 1,000 & $1,7^{-10}$ & & & & \\
\hline 7 & 1,000 & $7,8^{-14}$ & $<2,0^{-16}$ & $7,8^{-14}$ & $<2,0^{-16}$ & & & \\
\hline 8 & 0,004 & $8,9^{-10}$ & $<2,0^{-16}$ & $8,9^{-10}$ & $<2,0^{-16}$ & 0,004 & & \\
\hline 9 & $1,6^{-11}$ & 0,030 & $8,3^{-14}$ & 0,030 & $8,3^{-14}$ & $1,6^{-11}$ & 0,019 & \\
\hline 10 & $<2,0^{-16}$ & 0,007 & 0,038 & 0,007 & 0,034 & $<2,0^{-16}$ & $8,3^{-14}$ & $1,4^{-10}$ \\
\hline
\end{tabular}


Para os índices de Margalef e de Menhinick, os cenários 4 e 6 apresentaram os maiores valores médios estimados por unidade amostral (Tabela 2). Apesar disso, o cenário 6 não deve ser considerado como a melhor opção para o planejamento da composição florística da arborização de ruas porque contempla a utilização de 331 espécies arbóreas diferentes, distribuídas em 15 unidades amostrais (Tabela 1). Essa quantidade de espécies é um fator técnico indesejável por ser impraticável a execução de um plano de arborização de ruas com essas características. Por meio disso, seriam mais limitantes as possibilidades de produção das mudas e a adoção de práticas de manutenção adequadas, principalmente a operacionalização da poda (época de execução, maquinário e tolerância à poda). Entretanto, para o cenário 4 obteveram-se valores significativamente iguais ao cenário 6 , quando considerada a média dos valores entre as unidades amostrais.

Comparando-se a Tabela 4 com a Tabela 2, verifica-se que o índice de Simpson tende a apresentar um valor menor quanto maior o valor de riqueza específica, ao passo que o índice de McIntosh apresenta tendência contrária. Essas observações ficam reforçadas pelas informações apresentadas na Tabela 5, na qual há indicação de que para as situações hipotéticas dos cenários planejados há correlação negativa entre o índice de Simpson e os índices de riqueza, e correlação positiva desses com o índice de McIntosh. Essa tendência inversa entre os índices de Simpson e de McIntosh também foi relatada por Schaaf et al. (2006) ao analisarem, por meio de índices

Tabela 4. Valores médios de cada índice de diversidade, por unidade amostral, observados em cada cenário de diversidade elaborado.

Table 4. Average values of each diversity index, per unit plot, observed in each diversity scenario created.

\begin{tabular}{|c|c|c|c|c|c|c|c|c|c|c|}
\hline \multirow{2}{*}{$\begin{array}{c}\text { Índices de } \\
\text { Diversidade }\end{array}$} & \multicolumn{10}{|c|}{ Cenários de diversidade testados } \\
\hline & 1 & 2 & 3 & 4 & 5 & 6 & 7 & 8 & 9 & 10 \\
\hline Simpson & 1,00 & 0,50 & 0,12 & 0,06 & 0,12 & 0,06 & 0,12 & 0,06 & 0,15 & 0,07 \\
\hline McIntosh & 0 & 0,31 & 0,68 & 0,78 & 0,68 & 0,78 & 0,29 & 0,51 & 0,65 & 0,76 \\
\hline Shannon & 0,18 & 0,69 & 2,21 & 2,89 & 2,21 & 2,89 & 2,21 & 2,89 & 1,97 & 2,66 \\
\hline Bulla & --- & 0,98 & 0,70 & 0,71 & 0,70 & 0,71 & 0,83 & 0,86 & 0,80 & 0,81 \\
\hline Pielou & --- & 1,00 & 0,91 & 0,94 & 0,91 & 0,94 & 3,19 & 2,08 & 0,95 & 0,96 \\
\hline
\end{tabular}

Tabela 5. Correlação entre as estimativas dos índices de diversidade obtidos a partir dos cenários de diversidade (Cen) e a partir dos valores totais dos dados do inventário da arborização de ruas (Inv), com respectivas significâncias estatísticas ( $p$-valor).

Table 5. Correlation between estimative of diversity indexes obtained from diversity scenarios (Cen) and from total values of tree-lined street inventory (Inv) with respective statistical significances ( $p$-valor).

\begin{tabular}{|c|c|c|c|c|c|c|c|c|c|c|c|c|}
\hline & \multicolumn{2}{|c|}{ Margalef } & \multicolumn{2}{|c|}{ Menhinick } & \multicolumn{2}{|c|}{ Simpson } & \multicolumn{2}{|c|}{ McIntosh } & \multicolumn{2}{|c|}{ Bulla } & \multicolumn{2}{|c|}{ Pielou } \\
\hline & Cen & Inv & Cen & $\overline{\text { Inv }}$ & Cen & Inv & Cen & Inv & Cen & Inv & Cen & Inv \\
\hline Menhinick & 0,98 & 0,96 & & & & & & & & & & \\
\hline$p$-valor & 0 & 0 & & & & & & & & & & \\
\hline Simpson & $-0,71$ & $-0,64$ & $-0,67$ & $-0,73$ & & & & & & & & \\
\hline$p$-valor & 0 & $-0,011$ & 0 & $-0,002$ & & & & & & & & \\
\hline McIntosh & 0,82 & 0,57 & 0,79 & 0,69 & $-0,98$ & $-0,98$ & & & & & & \\
\hline$p$-valor & 0 & $-0,027$ & 0 & $-0,004$ & 0 & 0 & & & & & & \\
\hline Bulla & $-0,25$ & 0,21 & $-0,24$ & 0,34 & $-0,23$ & $-0,7$ & 0,15 & 0,75 & & & & \\
\hline$p$-valor & $-0,066$ & $-0,448$ & $-0,077$ & $-0,216$ & $-0,097$ & $-0,004$ & $-0,274$ & $-0,001$ & & & & \\
\hline Pielou & 0,1 & 0,35 & 0,1 & 0,5 & $-0,65$ & $-0,88$ & 0,56 & 0,92 & 0,82 & 0,93 & & \\
\hline$p$-valor & $-0,454$ & $-0,198$ & $-0,472$ & $-0,06$ & 0 & 0 & 0 & 0 & 0 & 0 & & \\
\hline Shannon & 0,92 & 0,88 & 0,89 & 0,91 & $-0,9$ & $-0,91$ & 0,97 & 0,87 & $-0,01$ & 0,57 & 0,38 & 0,73 \\
\hline$p$-valor & 0 & 0 & 0 & 0 & 0 & 0 & 0 & 0 & $-0,980$ & $-0,026$ & $-0,004$ & 0,002 \\
\hline
\end{tabular}


de diversidade, as modificações florístico-estruturais de um remanescente de Floresta Ombrófila Mista Montana no período entre 1979 e 2000.

A tendência de correlação para esses índices também foi observada para os valores reais do inventário da arborização de ruas realizado no ano de 2010 (Tabela 5).

Tanto para os índices de dominância quanto para os índices de equidade testados não foi constatada influência significativa do espaçamento planejado sobre os valores dos índices ( $\mathrm{GL}=5 ; p$-valores $>0,01$ ), quando analisados os dados cenário a cenário. Para a análise comparativa entre os valores de diversidade dos cenários foi constatada diferença significativa $(\mathrm{GL}=8$; $p$-valores $<0,01)$.

Em relação ao índice de Bulla, constatou-se que os cenários 1 e 2 apresentaram os maiores valores, seguidos dos cenários 9 e 10, quando considerado o valor total das 15 unidades amostrais objeto dos cenários. Se considerados os valores médios, o cenário 2 sobressai com a maior equidade, seguido dos cenários 8 e 7 . Em relação ao índice de Pielou, constatou-se que os cenários 7 e 8 apresentaram os maiores valores, seguidos dos cenários 1 e 2 , quando considerado o valor total das 15 unidades amostrais objeto dos cenários. Se considerados os valores médios, o cenário 7 se sobressai com a maior equidade, seguido pelos cenários 8 e 2 . As variações constatadas para os índices de Bulla e de Pielou se devem ao número de ruas existentes em cada unidade amostral e ao comprimento delas dentro da área amostrada (o que pode adicionar mais ou menos árvores na amostragem total), além da quantidade de espécies e repetição delas na composição dos cenários.

De acordo com Bobrowski \& Biondi (2012), deve-se conciliar a diversificação de espécies e a uniformidade na composição da arborização de ruas, a fim de propiciar maior gama de benefícios possíveis e de facilitar a operacionalização das práticas de manejo necessárias. Além disso, uma melhor equidade entre as espécies que compõem a arborização de ruas de uma amostra, de um bairro e da cidade é um fator interessante porque expressa e ressalta atributos estéticos da composição florística como o ritmo, a textura, a cor, a forma, o equilíbrio, o contraste, dentre outros.
Da mesma forma que para os índices de riqueza específica, a análise comparativa de valores de índices de equidade de trabalhos como os de Souza (2012) e Santos et al. (2013) pode ser problemática se não há clareza na descrição da área total amostrada ou se há mistura entre as informações obtidas em praças, parques e na arborização de ruas.

Richter et al. (2012), ao analisarem a arborização da cidade de Mata, RS, encontraram um valor igual a 0,70 para o índice de Pielou e concluíram que havia boa uniformidade na composição da flora avaliada. A consideração de que a equidade é boa deve ser avaliada com cautela, pois o resultado desses índices depende muito de como está composta a arborização das ruas analisadas. Pode haver elevada uniformidade entre as espécies, porém elas podem estar distribuídas de forma dispersa pelas unidades amostrais e muitas vezes correspondem a plantios voluntários que simplificam o efeito estético das árvores, diluindo-o. O controle da composição por meio de cenários de diversidade pode auxiliar na obtenção de valores ideais de uniformidade para comprimento de calçadas, número de ruas, de árvores e espécies específicas para um inventário da arborização de ruas, o que permite afirmar com mais respaldo se a uniformidade observada é boa ou ruim.

Por meio da análise do índice de Shannon-Wiener, a partir dos dados totais das unidades amostrais constatou-se que os maiores valores de diversidade foram obtidos para os cenários 6 e 10, os quais também apresentaram maior riqueza específica na composição florística. Porém, quando analisado o índice a partir dos valores médios (Tabela 4), os cenários 4,6 e 8 apresentaram os maiores valores. $\mathrm{Na}$ análise cenário a cenário não foi constatada influência significativa do espaçamento sobre os valores do índice $(\mathrm{GL}=5$; $p$-valores $>0,01)$, porém entre os valores obtidos para os cenários concebidos a diferença foi significativa $(\mathrm{GL}=8$; $p$-valores $<0,01)$.

Para esse índice foi constatada forte correlação com os valores dos índices de riqueza específica e de dominância, mas também uma baixa correlação com os valores dos índices de equidade dos cenários planejados (Tabela 5). Essas tendências de correlação ficaram mais evidentes quando da decomposição do índice pela análise SHE. Para Magurran (2011), o 

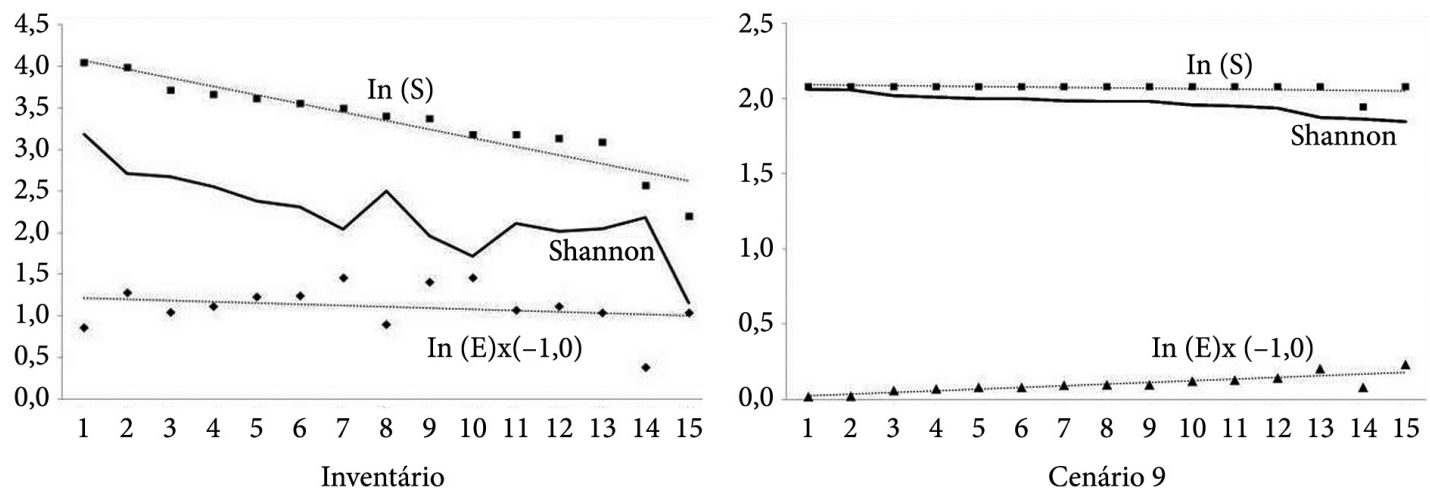

Figura 2. Variação do índice de Shannon-Wiener para os dados do inventário da arborização de ruas e do cenário 9 de diversidade, com linhas de tendência dos componentes riqueza específica $[\ln (S)]$ e uniformidade $[\ln (E)]$.

Figure 2. Variation of Shannon-Wiener index for data of tree-lined street inventory and of scenario 9 of diversity, with trend lines of the components species richness $(\ln (S))$ and uniformity $(\ln (E))$.

índice de Shannon-Wiener é influenciado mais pela riqueza de espécies, entretanto um aumento no valor do índice pode ser decorrente de um aumento na riqueza de espécies, na uniformidade de composição ou de ambas as características. Cowett \& Bassuk (2014) observaram acentuada correlação entre os valores de Shannon-Wiener e a riqueza de espécies e pequena correlação com a equidade de distribuição das espécies.

Na Figura 2 se demonstra que o índice de ShannonWiener apresenta redução no seu valor à medida em que diminui a riqueza específica (quantidade de espécies nas amostras), sendo a sua composição influenciada de forma mais proeminente por essa componente para os dados do inventário da arborização de ruas. As variações verificadas nas curvas das componentes riqueza específica e uniformidade se devem ao descontrole da composição da arborização de ruas, por haver a contribuição voluntária da população com a introdução de espécies diferentes, além do plantio executado pela prefeitura municipal. Isso reduz a uniformização da composição florística da arborização de ruas por diluir a quantidade de árvores em maior número de espécies e de maneira desproporcional.

Por outro lado, para os dados de diversidade do cenário 9 constatou-se que os valores desse índice diminuem à medida em que aumentam os valores da componente uniformidade, sendo que a componente riqueza específica não manifestou alterações expressivas. A estabilidade verificada para a curva da componente riqueza específica se deve à manutenção (controle) da quantidade de espécies em cada uma das parcelas utilizadas na concepção do cenário. Já a variação da curva da componente uniformidade se deve às características de comprimento das ruas dentro de cada amostra e à quantidade delas, as qual é variável.

Comparando-se os dados do inventário com os dados do cenário 9 (Figura 3), verifica-se que as maiores reduções para o índice de ShannonWiener são constatadas para as unidades amostrais que apresentam os maiores valores de riqueza específica pelos índices de Margalef e de Menhinick. Isso reforça a afirmação de que o índice de Shannon é mais influenciado pela riqueza de espécies do que pela equidade, pois, na composição dos cenários, a riqueza específica foi controlada e uniformizada para cada unidade amostral.

Também se pode perceber que para os dados reais a diversidade de espécies (riqueza específica) foi acentuadamente maior em relação aos dados dos cenários 4 e 9. Para a uniformidade, os valores dos dados reais foram acentuadamente menores em relação aos cenários em comparação, por conta da composição florística menos regular. 

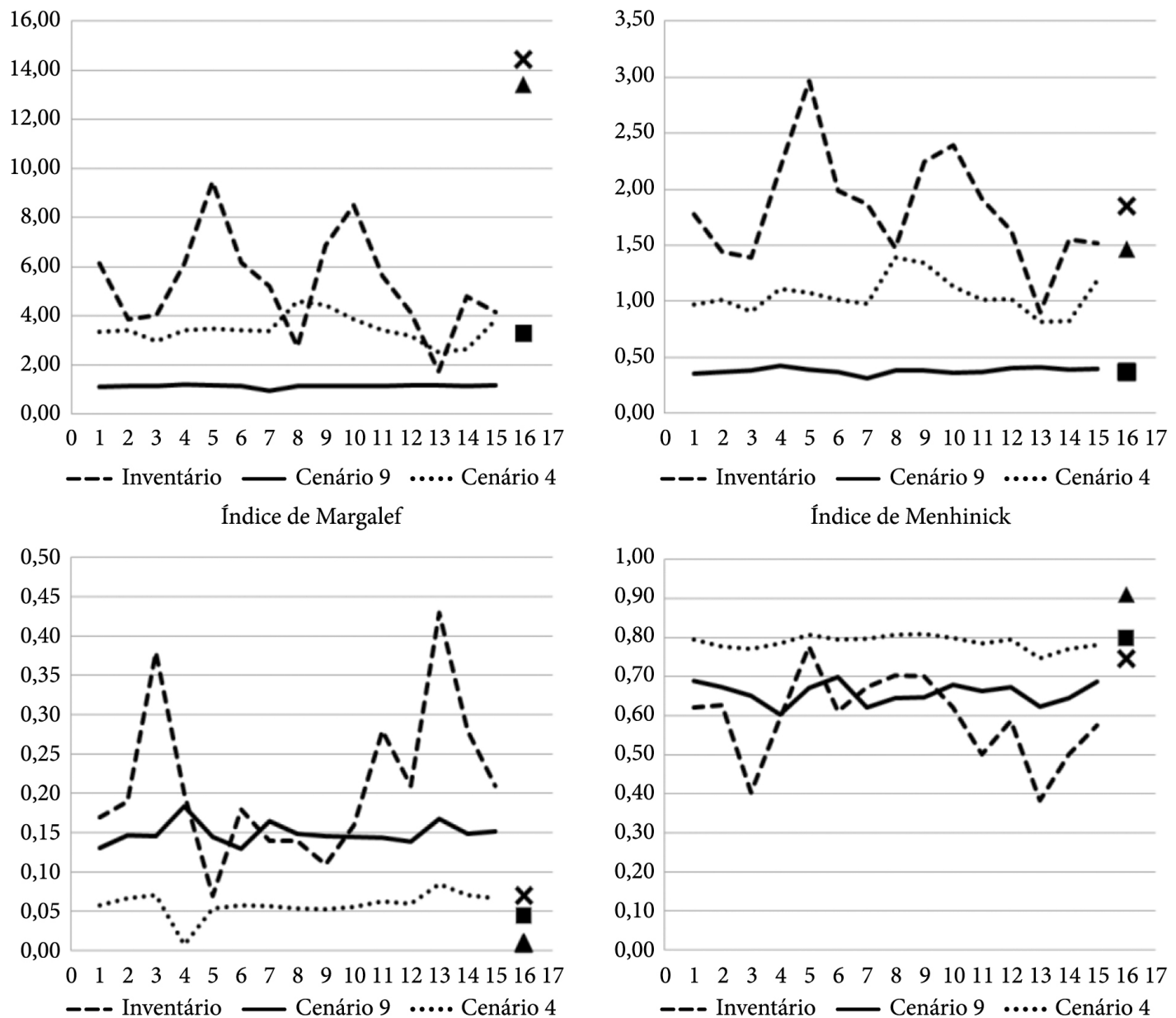

Índice de Simpson

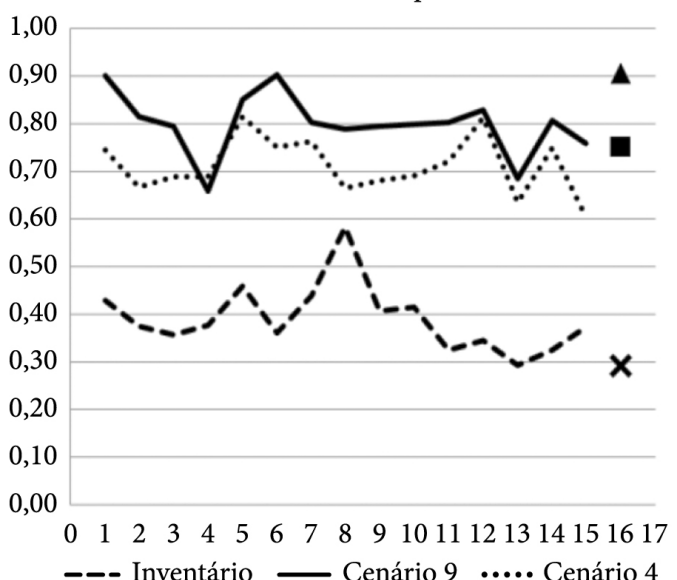

Índice de McIntosh

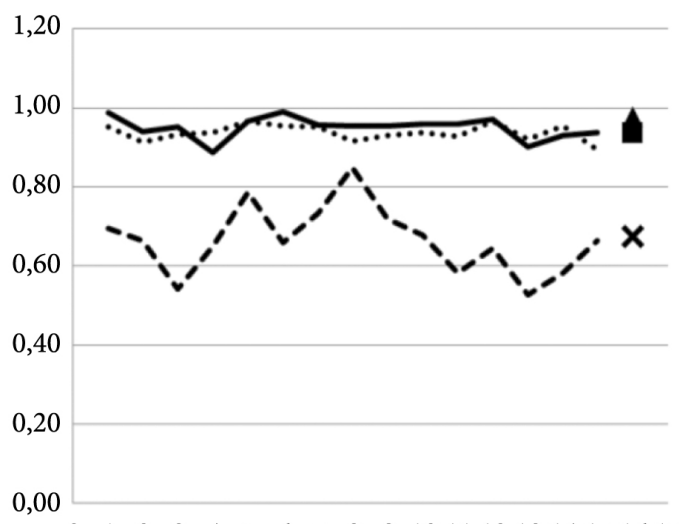

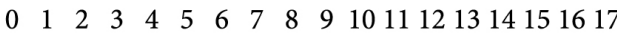

Índice de Bulla

Índice de Pielou

Figura 3. Variação da estimativa dos índices de diversidade para as 15 unidades amostrais e para os dados totais do inventário da arborização de ruas $(\times)$ do cenário $9(\boldsymbol{\Delta})$ e do cenário 4 de diversidade (•).

Figure 3. Estimative variation of diversity indexes for the fifteen unit plots and for the total data of the tree-lined street inventory $(\times)$, of the scenario $9(\boldsymbol{\Delta})$ and of scenario 4 of diversity $(\mathbf{})$. 


\section{CONCLUSÕES}

Os espaçamentos de plantio planejados influenciaram significativamente os valores dos índices de Margalefe de Menhinick quando considerados os valores obtidos para as unidades amostrais.

Nas condições reais de plantio na arborização de ruas, a diversidade de composição tendeu a se expressar com maior riqueza específica e menor uniformidade em relação a uma situação planejada a partir de informações dos valores médios das variáveis que interferem na composição (espaçamento, quantidade de árvores e quantidade de espécies).

Os limites de diversidade (riqueza específica ou uniformidade) obervados em cenários de composição planejados podem servir como parâmetro técnico para avaliação da qualidade da informação encontrada nos inventários da arborização de ruas. Por meio desses cenários se obtém a composição mínima possibilitada pelas limitações impostas pela estrutura urbana (tamanho de calçada, distância das esquinas, distância dos postes, distância das guias rebaixadas, dentre outras), as quais afetam o número de árvores e o tipo de espécie que pode ser implantada nas calçadas.

\section{STATUS DA SUBMISSÃO}

Recebido: 18 maio, 2015

Aceito: 25 out., 2015

\section{AUTOR(ES) PARA CORRESPONDÊNCIA}

\section{Rogerio Bobrowski}

Universidade Estadual do Centro-Oeste UNICENTRO, Rodovia BR 153, Km 7, CEP

84500-000, Irati, PR, Brasil

e-mail: bobrowski_roger@yahoo.com.br

\section{REFERENNCIAS}

Biondi D, Leal L. Caracterização das plantas produzidas no Horto Municipal da Barreirinha, Curitiba/PR. RevSbau 2008; 3(2): 20-36.

Bobrowski R, Biondi D. Caracterização do padrão de plantio adotado na arborização de ruas de Curitiba, Paraná. RevSbau 2012; 7(3): 20-30.

Bobrowski R. Estrutura e dinâmica da arborização de ruas de Curitiba, Paraná, no período 1984-2010 [dissertação].
Curitiba: Setor de Ciências Agrárias, Universidade Federal do Paraná; 2011.

Cowett FD, Bassuk NL. Statewide assessment of street trees in New York State, USA. Urban Forestry \& Urban Greening 2014; 13(2): 213-220. http://dx.doi.org/10.1016/j. ufug.2014.02.001.

Galvin MF. A methodology for assessing and managing biodiversity in street tree populations: a case study. Journal of Arboriculture 1999; 25(3): 124-128.

Insernhagen I, Le Bourlegat JMG, Carboni M. Trazendo a riqueza arbórea regional para dentro das cidades: possibilidades, limitações e benefícios. RevSbau 2009; 4(2): 117-138.

Jim CY, Chen WY. Diversity and distribution of landscape trees in the compact Asian city of Taipei. Applied Geography 2009; 29(4): 577-587. http://dx.doi.org/10.1016/j. apgeog.2009.01.002.

Kanieski MR, Araujo ACB, Longhi SJ. Quantificação da diversidade em Floresta Ombrófila Mista por meio de diferentes índices alfa. Scientia Forestalis 2010; 38(88): 567-577.

Kenney WA, Van Wassenaer PJE, Satel AL. Criteria and indicators for strategic urban forest planning and management. Arboriculture \& Urban Forestry 2011; 17(3): 108-117.

Magurran AE. Medindo a diversidade biológica. Curitiba: Editora da UFPR; 2011. Original em inglês.

Miranda TO, Carvalho SM. Levantamento quantitativo e qualitativo de indivíduos arbóreos presentes nas vias do bairro da Ronda em Ponta Grossa-PR. RevSbau 2009; 4(3): 143-157.

Moreno CE. Métodos para medir la biodiversidad. Zaragoza: M\&T Manuales y Tesis; 2001.

Nagendra H, Gopal D. Street trees in Bangalore: density, diversity, composition and distribution. Urban Forestry \& Urban Greening 2010; 9(2): 129-137. http://dx.doi. org/10.1016/j.ufug.2009.12.005.

Pohlert T. The Pairwise Multiple Comparison of Mean Ranks Package (PMCMR) [online]. Vienna: R Package; 2014 [citado 2015 jan. 20]. Disponível em: http://CRAN.Rproject.org/package $=\mathrm{PMCMR}$

R Development Core Team. R: a language and environment for statistical computing [online]. Vienna: R Foundation for Statistical Computing; 2014 [citado 2015 jan. 20]. Disponível em: http://www.R-project.org/

Raupp MJ, Cumming AB, Raupp EC. Street tree diversity in eastern north America and its potential for tree loss to exotic borers. Arboriculture \& Urban Forestry 2006; 32(6): 297-304.

Richards NA. Reasonable guidelines for street tree diversity. Journal of Arboriculture 1993; 19(6): 344-350. 
Richter C, Peiter MX, Robaina AD, Souza ARC, Ferraz RC, David AF. Levantamento da arborização pública de Mata/RS. RevSbau 2012; 7(3): 88-96.

Santos EM, Silveira BD, Souza AC, Schmitz V, Silva AC, Higuchi P. Análise quali-quantitativa da arborização urbana em Lages, SC. Revista de Ciências Agroveterinárias 2013; 12(1): 59-67.

Schaaf LB, Figueiredo A Fo, Galvão F, Sanquetta CR, Longhi SJ. Modificações florístico-estruturais de um remanescente de Floresta Ombrófila Mista Montana no período entre 1979 e 2000. Ciência Florestal 2006; 16(3): 271-291.

Siegel S, Castellan NJ. Estatística não-paramétrica para ciências do comportamento. Porto Alegre: Artmed; 2006.

Silva DF Fo, Bortoleto. Uso de indicadores de diversidade na definição de plano de manejo da arborização viária de Águas de São Pedro-SP. Árvore 2005; 29(6): 973-981. http://dx.doi.org/10.1590/S0100-67622005000600017.

Sjöman H, Östberg J, Bühler O. Diversity and distribution of the urban tree population in ten major Nordic cities. Urban Forestry \& Urban Greening 2012; 11(1): 31-39. http://dx.doi.org/10.1016/j.ufug.2011.09.004.
Souza ARC. Diagnóstico ambiental e paisagístico da arborização urbana do bairro centro de Santiago/RS [dissertação]. Santa Maria: Centro de Ciências Rurais, Universidade Federal de Santa Maria; 2012.

Sreetheran M, Adnan M, Khairil Azuar AK. Street tree inventory and tree risk assessment of selected major roads in Kuala Lumpur, Malaysia. Arboriculture \& Urban Forestry 2011; 37(5): 226-235.

Subburayalu S, Sydnor TD. Assessing street tree diversity in four Ohio communities using the weighted Simpson index. Landscape and Urban Planning 2012; 106(1): 4450. http://dx.doi.org/10.1016/j.landurbplan.2012.02.004.

Teixeira IF, Silva RM, Tatsch GL. Compatibilidade da arborização de ruas da Avenida Celestino Cavalheiro, São Gabriel-RS. Floresta e Ambiente 2011; 18(4): 438-450. http://dx.doi.org/10.4322/floram.2011.063.

Thompson JR, Nowak DJ, Crane DE, Hunkins JA. Iowa, U.S., communities benefit from a tree planting program: characteristics of recently planted trees. Journal of Arboriculture 2004; 30(1): 1-10. 